\title{
The Role of Endoscopic Ultrasound in Pancreatic Disorders
}

\author{
William R. Brugge \\ Gastrointestinal Unit, Massachusetts General Hospital, Boston, MA
}

Key Words: Endoscopic ultrasound; pancreatitis; pancreatic cancer; cystic neoplasms; chronic pancreatitis.

\section{Introduction}

Endoscopic ultrasound (EUS) represents one of the major advances in gastrointestinal endoscopy in the past 10 years. Originally designed to image mass lesions in the esophagus and stomach, the technique has been increasingly applied to a wide range of gastrointestinal disorders. Since the pancreas lies in such close proximity to the stomach and duodenum, imaging of the pancreas has become particularly successful.

EUS imaging of the upper gastrointestinal tract has become possible with the development of small ultrasound probes that can be placed in the esophagus and stomach. Most commonly, EUS probes are located on the tips of endoscopes, and imaging of the pancreas is possible from locations in the duodenum and stomach. The frequency of the ultrasound waves emitted and received by an ultrasound probe determines the depth of penetration and resolution; highfrequency waves produce high-resolution images with a shallow tissue penetration and low-frequency waves result in lower-resolution images. For optimal imaging of the pancreatic-biliary system, midrange

*Address to which all correspondence and reprint requests should be sent: William R. Brugge, 127 Bulfinch Building, GI Unit, Massachusetts General Hospital, Boston, MA 02114. E-mail: Brugge.William@mgh.harvard.edu frequencies are often used, usually $5-7.5 \mathrm{MHz}$. At these frequencies, structures within approx $7 \mathrm{~cm}$ of the stomach and duodenum are well seen, including the entire pancreas, the branches of the portal venous system, and the extrahepatic biliary system. Higherfrequency imaging provides high-resolution imaging of the structures, such as the ampulla of Vater, that are within $1-2 \mathrm{~cm}$ of the probe.

There are two basic designs for endosonoscopes, radial imaging (GIF UM-20 Olympus, Inc., Lake Success, NY) and curved linear array imaging (FG32UA, Pentax, Inc., Orangeburg, NY). The Olympus radial instrument operates at 12 and 7.5 $\mathrm{MHz}$. The Pentax unit operates at 5 and $7.5 \mathrm{MHz}$ with the capability of using color Doppler and pulse Doppler ultrasound with the Hitachi ultrasound console (1). The curved linear array design also makes it possible to direct needle aspiration biopsies, an important feature for the management of patients with pancreatic masses.

Optimal EUS imaging of the pancreas and its associated structures is dependent on the placement of the EUS probe in close proximity to the area of interest. Pancreatic head lesions are best seen from the first and second portions of the duodenum; body lesions are best seen from the stomach; and tail lesions can be optimally imaged from the fundus of the stomach. Although technically there are few difficulties in optimal probe placement in the stomach and 
duodenum, the interpretation of images representing the retroperitonium requires considerable experience.

EUS has been used to provide detailed imaging of benign and malignant diseases of the pancreas. Past studies have concentrated on the ability of EUS to detect malignant masses, and more recently, EUS has been used to stage and biopsy malignant and premalignant disorders of the pancreas.

\section{Malignant Diseases}

The EUS features of pancreatic adenocarcinoma have been well defined (1-3). The irregular, hypoechoic masses are easily identified within the homogenous parenchyma of the pancreas (Fig. 1). EUS is capable of providing detailed imaging of the interiors, borders, and location of the masses. The interiors of the masses are often heterogeneous and contain hypoechoic, somewhat cystic regions of the tumor that probably reflect central necrosis. The main pancreatic duct is often dilated as a result of compression and obstruction by the infiltrating mass, which usually involves the main pancreatic duct. The external borders of a malignant mass are often irregular, particularly in the pancreatic parenchyma, where the infiltrative nature of the masses is readily appreciated. Although the borders are irregular, the distinction between malignant and nonmalignant pancreatic tissue can be easily made. Small masses, $<3 \mathrm{~cm}$ in diameter, are usually found in the head of the pancreas, surrounding the distal intrapancreatic common bile duct. Most pancreatic head masses are within close proximity of the duodenum and are easily imaged with EUS. The location of early malignancies as seen by EUS may be a reflection of their clinical presentation, biliary obstruction, rather than a reflection of where pancreatic malignancies originate. Larger masses, $>3 \mathrm{~cm}$ in diameter, have often invaded out of the pancreas and extended posteriorly toward the branches of the portal venous system or superiorly along the bile duct and the duodenum.

The ability of EUS to detect pancreatic adenocarcinoma has been compared to several other imaging tests, including US, CT, MRI, and ERCP (4-6). Not surprisingly, EUS is much more sensitive than traditional transabdominal ultrasonography in detecting pancreatic masses, particularly early, intrapancreatic masses. The reasons for the supe-

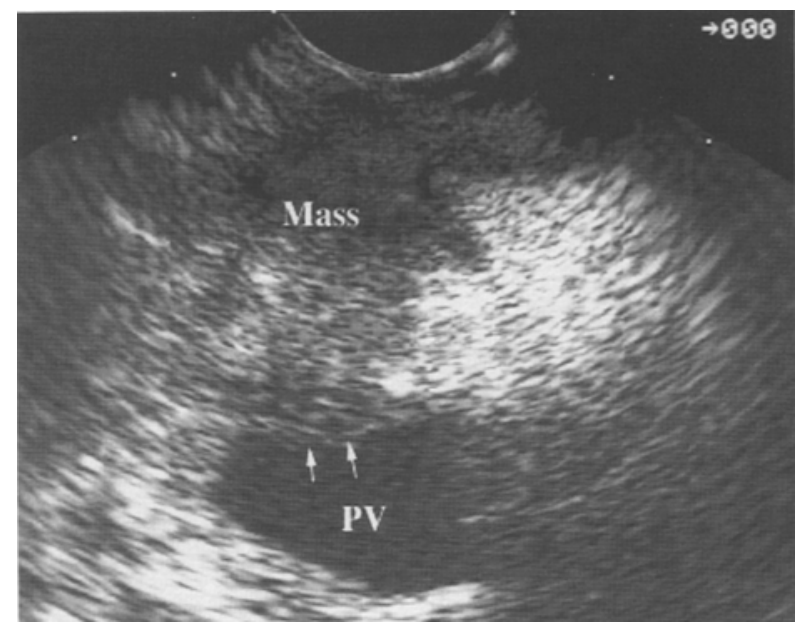

Fig. 1. Large irregular hypoechoic mass in the head of the pancreas invading one wall of the portal vein (PV). At surgery, the patient was found to have unresectable adenocarcinoma.

rior imaging with EUS include the lack of bowel gas between the pancreas and the probe, the ability to use high-frequency ultrasound, and the close proximity between the probe and the pancreas. Although EUS has not been compared to spiral CT scanning, EUS has been shown to be more sensitive and more specific than traditional dynamic CT scanning $(2,7,8)$. EUS is particularly effective at detecting small $(<2 \mathrm{~cm})$ intrapancreatic masses that have not deformed the contour of the gland, a finding that is often used for making the diagnosis of a pancreatic mass with CT scanning.

The use of MRI in the diagnosis of pancreatic cancer has been studied and compared to EUS in several studies. In general, MRI has not demonstrated good sensitivity for the detection of small pancreatic masses. Recently, in a direct comparison among MRI, CT, and EUS, MRI was found to be less sensitive than EUS (93 vs $67 \%$ ) for detecting a malignant pancreatic mass (7). Both imaging modalities, however, were $100 \%$ specific for predicting the presence of a malignant pancreatic mass.

Traditionally, ERCP has been the most sensitive test for pancreatic cancer. Although EUS may be more of a complementary test, there have been trials comparing the ability of EUS and ERCP at detecting and diagnosing pancreatic cancer (4). In a comparison of EUS, ERCP, CT, and US, Rösch found that EUS had a sensitivity of $99 \%$ in detecting pancreatic 
cancer in 132 patients with suspected pancreatic cancer. All other imaging tests had lower sensitivities: US (67\%), CT (77\%), and ERCP (90\%). However, EUS was accurate in differentiating between pancreatitis and malignancy in only $76 \%$ of patients. The use of EUS in diagnosing pancreatic cancer has two advantages, lack of invasiveness and the ability of EUS to stage and localize the mass. The major advantage of ERCP is its ability to provide therapeutic biliary drainage at the time of the diagnostic testing.

The specificity of EUS imaging of malignant pancreatic lesions has not been as well studied as the sensitivity of EUS (9). The most common EUS finding in pancreatic cancer is a hypoechoic parenchymal mass. The differential diagnosis of this finding is early pancreatic cancer or focal pancreatitis. Despite many attempts to develop criteria that might differentiate between cancer and pancreatitis, there are no ultrasonographic findings that are specific for malignancy. Most of the prominent EUS features that are readily imaged in pancreatic cancer, such as the finding of an infiltrating hypoechoic mass, can also be seen in focal, acute, or chronic pancreatitis. Palazzo and coworkers reported in their series four false-positive EUS imaging results in 64 patients with suspected pancreatic cancers $(10)$.

\section{Staging of Pancreatic Cancer}

In contrast to the lack of diagnostic EUS criteria for pancreatic cancer, a wide range of staging criteria have been developed for pancreatic cancers. Many EUS staging criteria for pancreatic cancer use tumor staging systems (11). The TNM staging classification has been used to define whether a pancreatic mass is within the pancreas or has extended to adjacent structures (12). T1 masses, although rarely seen, are completely intrapancreatic and do not involve the intrapancreatic bile duct or duodenal wall. T2 masses are the most commonly encountered malignancy that can be surgically resected, and they may involve the distal common bile duct or the wall of the duodenum. The most common EUS finding that defines extrapancreatic extension of malignancy is tumor invasion of the branches of the portal venous system. The main portal vein is the vein that is most commonly involved in pancreatic cancer and is easiest to define with EUS (Fig. 1). Body and tail masses often involve the splenic vein that courses in close proximity to the entire length of the pancreas (13). The superior mesenteric vein is the least accessible to EUS imaging and is technically more difficult to image than the portal vein (Fig. 2). The overall accuracy of EUS in correctly staging pancreatic masses was $92 \%$ in Tio et al.'s series of 43 patients with pancreatic cancer (12). Lymph node involvement was correctly predicted in $91 \%$ of patients.

There have been several large studies that have defined various EUS criteria for diagnosing malignant invasion of the branches of the portal venous system. In general, the highest accuracy rates for predicting malignant venous invasion have been achieved by using a combination of ultrasonographic criteria. Initially, Yasuda et al. used a combination of two ultrasound findings that represented tumor invasion of the wall of the portosplenic veins and compression of the venous lumen (14). Similarly, "close contact," "abnormal contour," and "loss of hyperechoic interface" were three EUS criteria that were used by Rösch to achieve an accuracy rate of $94 \%$ (15). The highest accuracy rate for vascular staging by EUS was achieved by combining the findings of "venous collaterals," "tumor in lumen," and "loss of interface" in staging of pancreatic cancer (16). Recently, Nakaizumi has used a similar combination of criteria with an accuracy rate of $96 \%$ for predicting malignant venous invasion (8). Brugge et al. reported the use of four separate EUS criteria and found that the use of the finding "irregular venous wall" achieved the highest accuracy rate, $87 \%$, for all branches of the portal venous system (17).

If EUS is to have a major role in the staging of pancreatic cancer, it will be critical to compare its accuracy rate with those results obtained by mesenteric angiography. There have been three prospective comparison trials between EUS and angiography in the vascular staging of pancreatic cancers. In Rösch et al.'s trial, EUS was found to be twice as accurate as mesenteric angiography because of the ability of EUS to diagnose early invasion of the portal vein wall (15). In two other studies, the accuracy rates were similar with both modalities. Although Brugge et al. found EUS and angiography to have comparable accuracy ( 87 vs $85 \%$ ) for predicting vascular invasion at surgery, EUS was particularly good for portal vein invasion, whereas angiography excelled at diagnosing superior mesenteric vein inva- 


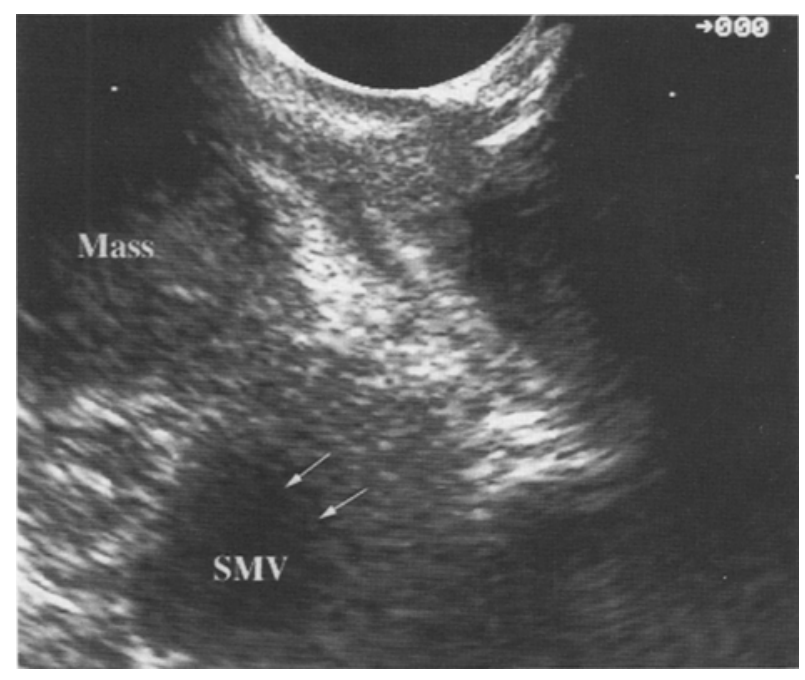

Fig. 2. Pancreatic mass $(2 \times 3 \mathrm{~cm})$ surrounding the superior mesenteric vein (SMV) and invading one wall (arrows). The invasion of the SMV was confirmed at surgery and with angiography.

sion (17). Mukai has also reported similar results with EUS, demonstrating vascular invasion accurately in $77 \%$ as compared to $73 \%$ with angiography (18).

Another strategy in the vascular staging of pancreatic cancers is the use of duplex Doppler ultrasonography (19). The Pentax endosonoscope has the capability of providing pulse and color Doppler studies of the portal and splenic veins. Abnormal pulsed Doppler tracings suggestive of encasement of the portal venous system have been reported to be predictive of unresectability. In addition, EUS may provide convincing evidence of portosplenic venous thrombosis (20). This type of thrombosis may be associated with a pancreatic mass, chronic pancreatitis, or tumorous invasion through the wall of the vein (21).

Historically, nearly all studies of the ability of EUS to detect and stage pancreatic masses have been performed using radial imaging EUS instruments. Recently, linear array instruments have been used and offer the advantage of being capable of directing needle aspiration biopsies of the pancreas. Radial imaging provides images of the retroperitoneum in cross-sectional orientation, similar to CT scanning. Linear array imaging is oriented along a $100^{\circ}$ sagittal section, parallel to the long axis of the endoscope. In comparison with radial imaging, linear imaging is more difficult to learn and interpret. However, a com- parison between the staging abilities of these two types of EUS imaging modalities has demonstrated similar sensitivities, specificities, and accuracy rates for staging pancreatic cancer (22).

The design of linear array EUS instruments affords the possibility of ultrasonography-guided needle aspiration biopsies. A 23-gage stainless-steel needle with a beveled stylet has been designed for the Pentax endosonoscope $(23,24)$. Pancreatic masses are biopsied by passing the needle through the wall of the stomach or duodenum and into the adjacent pancreatic tissue (Fig. 3). Once in the mass, the stylet is removed, and tissue is aspirated from the periphery of the mass and adjacent nodes. EUS-directed needle aspiration biopsies of pancreatic masses have recently been described in several series in which the authors have collected their overall experience with this new technique. In two separate reports, Wiersema et al. and Cheng et al. in the US have reported their experience with a total of 16 patients with pancreatic lesions who had successful needle aspiration biopsies $(25,26)$. Overall, the sensitivity was $91 \%$ in making a diagnosis of malignant disease. More recently, Giovannini et al. have described their 3-yr experience in needle aspiration biopsy, including 43 patients with pancreatic tumors (27). Although there were no false-positive results with needle aspiration biopsies of the pancreas, in 9 of the 43 patients, they could not obtain adequate tissue to make a tissue diagnosis of pancreatic malignancy. Peripancreatic lymph node biopsies were technically easier and the quality of the tissue superior. Liver metastases from pancreatic primaries can also be biopsied with EUS-guided needles using a transgastric approach. Malignant ascites have also been diagnosed by transgastric needle aspiration (28). There have been a couple of reports of radial imaging endosonoscopes that have guided a needle aspiration biopsy of the pancreas (29). Unfortunately, radial EUS imaging does not provide complete imaging of the course of the needle into the pancreas.

Wiersema et al. have recently reported that the use of EUS can reduce the cost of managing patients with pancreatic cancer by reducing the number of patients undergoing unnecessary attempted resections and providing pathologic proof of a malignancy with needle aspiration biopsies (30). Although there have been no head-to-head comparisons between CT- and EUS-guided aspiration biopsies of the pancreas, EUS 


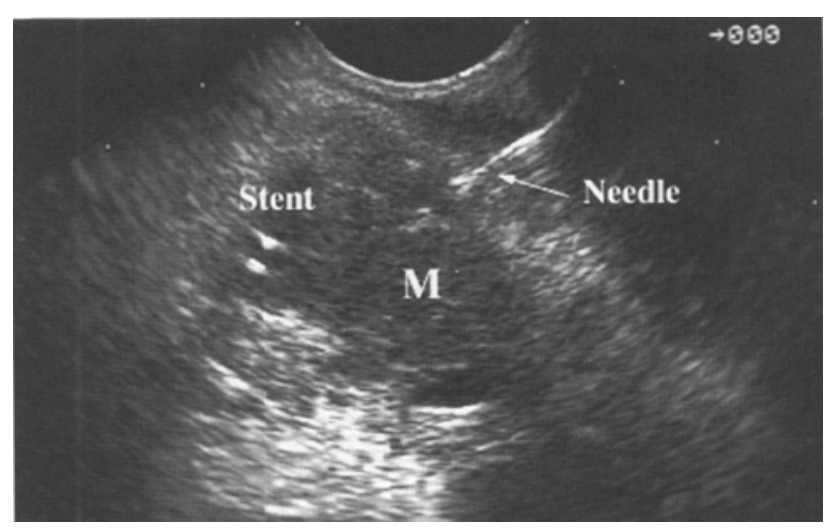

Fig. 3.EUS-directed needle biopsy of a pancreatic mass (M) surrounding the distal common bile duct. The distal common bile duct contains a stent that appears as two white lines.

might offer two advantages. Most importantly, EUSdirected biopsies are not complicated by the risk of peritoneal tumor seeding. Second, the placement of an aspiration needle into a small intrapancreatic mass should be more accurate with EUS imaging because of the high resolution of the EUS imaging.

The first step in therapeutic EUS has been taken with the demonstration of celiac ganglion neurolysis (31). Wiersema has reported his experience with this technique in patients with pancreatic cancer and has found the celiac ganglion injections produce significant reductions in pain from malignancy. He has used a combination of ethanol and bupivacaine in the injectant to provide immediate relief as well as long-lasting analgesic by interrupting afferent transmission in the sympathetics. The most common complications of the procedure were diarrhea and transient hypotension, probably a transient result of sympathetic blockade. The pain relief has been reported to be sustained over at least a 2-mo period. There have been no trials comparing EUS- and CTdirected celiac neurolysis.

\section{Cystic Lesions of the Pancreas}

EUS is particularly good at providing detailed imaging of cystic lesions of the pancreas (Fig. 4). Ultrasound imaging is not only very sensitive for detecting cystic lesions, but it can also examine the wall structure and details of the interior of the cysts. Pseudocysts appear as unilocular or multilocular

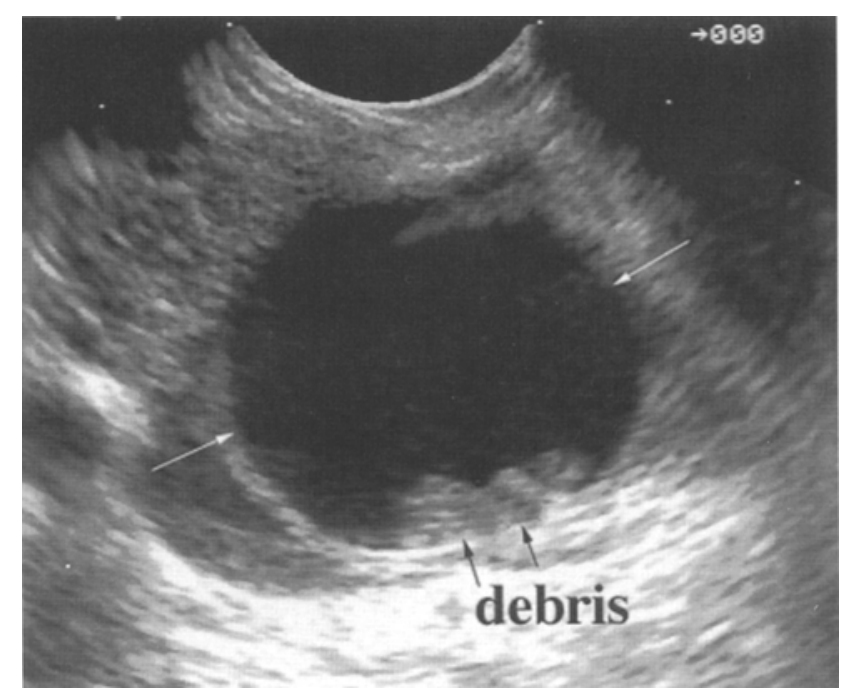

Fig. 4. A 3-cm thin-walled intrapancreatic pseudocyst in the head of the pancreas associated with chronic pancreatitis. A small amount of debris is seen along one wall.

fluid-filled structures with either thin or thick walls. Small pseudocysts, particularly intrapancreatic ones, can be easily examined in detail. EUS can determine the location of a pseudocyst in relationship to the wall of the duodenum or stomach, while also determining the thickness of the wall. These factors as well as whether a pseudocyst is simple or complex can help determine the best approach to drainage (32). Large pseudocysts are more difficult for EUS to assess since the entire border of the pseudocyst cannot be examined and its relationship to the upper GI tract may be difficult to describe. However, if a portion of the pseudocyst can be localized in relationship to the stomach, EUS can determine the thickness of the cyst-gastrostomy wall, and whether there are vascular structures in close proximity to a potential puncture and drainage site.

EUS-assisted drainage of pancreatic pseudocysts will become an important tool in the near future. Since EUS can provide detailed information regarding the size, location, and configuration of the pseudocyst, it is likely that these characteristics will be used by endoscopists to select patients who will readily respond to endoscopic drainage. For example, small, unilocular cysts that are directly adjacent to the stomach might be amenable to a one-step endoscopic drainage under direct EUS imaging (33). Large, complex pseudocysts containing internal 
debris might be better managed with surgery or radiologic drainage. Binmoeller et al. recently reported a large experience of endoscopic drainage of pseudocysts (34). EUS was not only used to localize and measure the wall thickness, but in some cases, they reported using the Pentax endosonoscope with the directed needle aspirator to eliminate the pseudocyst. In the future, large channel endosonoscopes will allow the direct placement of large-diameter stents and drains into pancreatic pseudocysts.

\section{Cystic Neoplasms}

Mucinous-secreting tumors of the pancreas and mucinous ductal ectasia (MDE) may be confused with pseudocysts because they have a similar clinical presentation and CT/US scanning may not be able to differentiate readily between the two. The EUS imaging of cystic neoplasms has not been reported in detail. Mucinous cyst adenomas often appear as complex cystic masses characterized by thick walls and irregular septations. There may also be solid components to the mass and calcifications in the wall (35). EUS can usually differentiate between cystadenomacarcinoma and MDE. The cystic masses in MDE are usually small, thin-walled, and without septations (Fig. 5). Although the cystic lesions in MDE are not true cysts, but rather dilated secondary ducts, occasionally the "cysts" grow to large diameters (36). Another important feature of MDE that is apparent on EUS is the presence of a dilated pancreatic duct that may contain intraductal mucin. The pancreatic parenchyma may be normal or atrophic, but should not be calcified or fibrotic. Since MDE is a premalignant lesion, EUS can also be used to look for focal masses that might represent a neoplasm.

Serous cystadenomas may have a characteristic appearance on EUS imaging (Fig. 6). The cystic masses usually appear as a collection of small, thinwalled cysts that contain fluid without debris. Serous cystadenomas are not associated with masses or abnormalities of the pancreatic ducts, although there may be central stellate calcifications. Although EUS has been reported to have a diagnostic accuracy rate of $84 \%$ for cystadenomas, EUS was only marginally better than CT in differentiating between serous and mucinous cystadenomas $(37,38)$. EUS-directed cyst aspiration and fluid analysis have also been used to differentiate between serous and mucinous cyst-

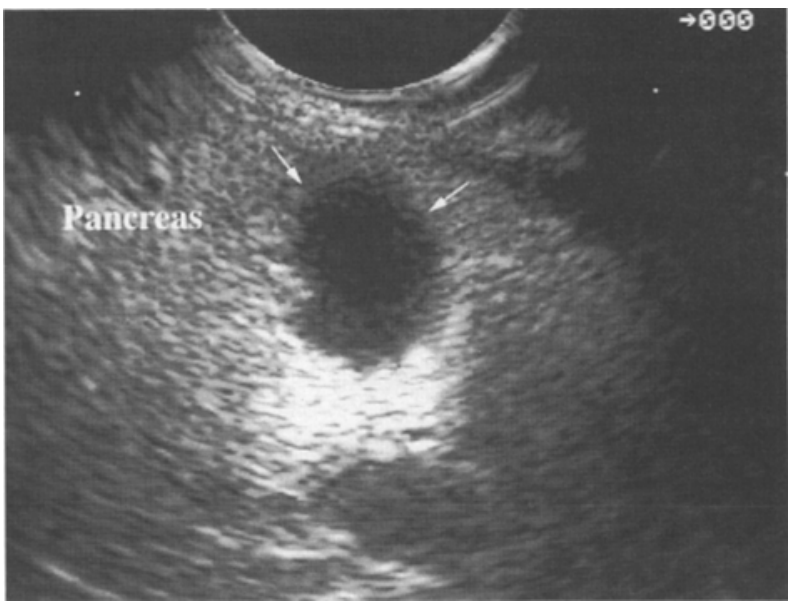

Fig. 5. A solitary pancreatic cyst (arrows) associated with mucinous ductal ectasia. Note the lack of a cyst wall. A dilated main pancreatic duct is seen behind the cyst. The pancreatic parenchyma is finely textured without evidence of fibrosis, fat, or calcifications.

adenomas, as well as pseudocysts. The fluid in serous cystadenomas is characterized by a low concentration of CEA, CA 19-9, and absence of mucins. In contrast, the mucinous cystadenoma carcinomas are characterized by high concentrations of CEA and mucins. EUS angiography, a new technique for assessing the vascularity of pancreatic masses, has been used to document vascularity of serous cystadenomas (39).

\section{Islet Cell Tumors}

Islet cell tumors, particularly insulinomas, can be well defined by EUS (Fig. 7). These small, often solitary masses appear as round, slightly hypoechoic masses within the pancreatic parenchyma (40). In contrast to adenocarcinomas, islet cell malignancies often have sharp, round borders and a homogenous texture. Occasionally, the small masses may be pedunculated and lie outside of the pancreas. The entire pancreas, from the uncinate process to the tail, must be carefully examined when evaluating a patient with a suspected islet cell malignancy, since the masses may be located anywhere in the gland. Fortunately, most islet cell tumors are unifocal and rarely metastasize.

The ability of EUS to detect and localize islet cell tumors has been well defined in several studies. In Rösch et al.'s series, the ability of EUS to localize 39 tumors (31 insulinomas, 7 gastrinomas, 1 glucago- 


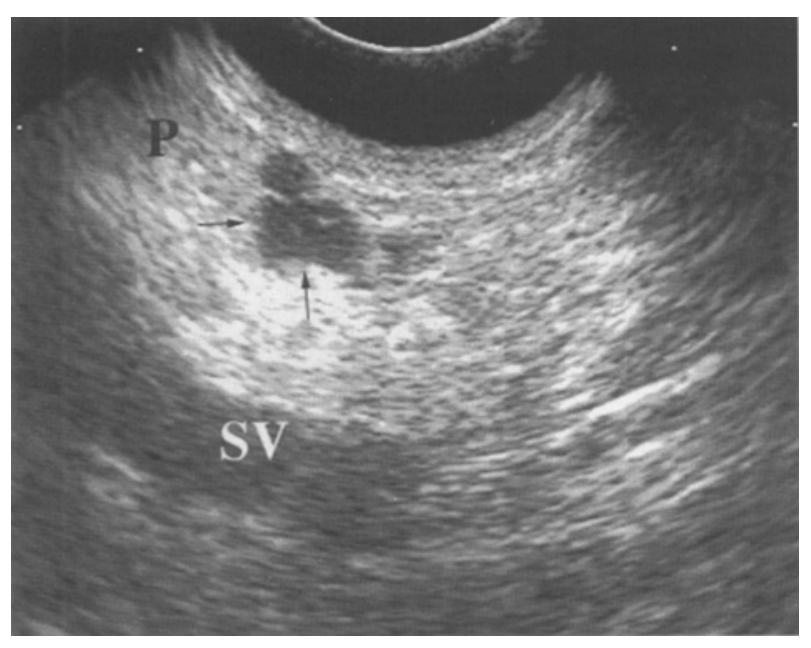

Fig. 6. An 8-mm thin-walled cystadenoma in the neck of the pancreas with the splenic vein (SV) behind the pancreas. Note the fine septations and hypoechoic interior, characteristic of a serous cystadenoma.

noma) in 37 patients who had nondiagnostic CT/US imaging was determined (41). EUS localized 32 of the 39 tumors $(82 \%)$ with a good correlation between EUS predicted size and surgical findings (0.5-2.5 $\mathrm{cm}$ diameter). Not only was CT/US not helpful in this series of patients, but angiography was also rather insensitive with a sensitivity rate of only $27 \%$. The specificity for EUS in this series of patients, $95 \%$, was calculated from those patients who ultimately were found not to have a tumor at surgery.

A compilation of recent studies of islet cell tumors revealed an accuracy rate of $89 \%$ for EUS (42). More recently, Zimmer et al. have compared the ability of EUS to localize neuroendocrine tumors with several advanced imaging techniques, including somatostatin receptor scintigraphy, CT, and MRI (43). EUS had the greatest sensitivity for tumor detection, $88 \%$, and was twice as sensitive as other technique for small $(<2 \mathrm{~cm})$ masses. EUS appeared to be capable of detecting small neuroendocrine masses whether they were intra- or extrapancreatic.

EUS has also been used to detect gastrinomas. Since more than $80 \%$ of gastrinomas lie within the "gastrinoma triangle,"EUS should be the ideal imaging technique. Ultrasonographically, gastrinomas are similar in appearance to islet cell tumors and can be localized within the pancreatic parenchyma (44). In a recent prospective comparison of EUS, CT, and angiography in 22 patients with surgically proven

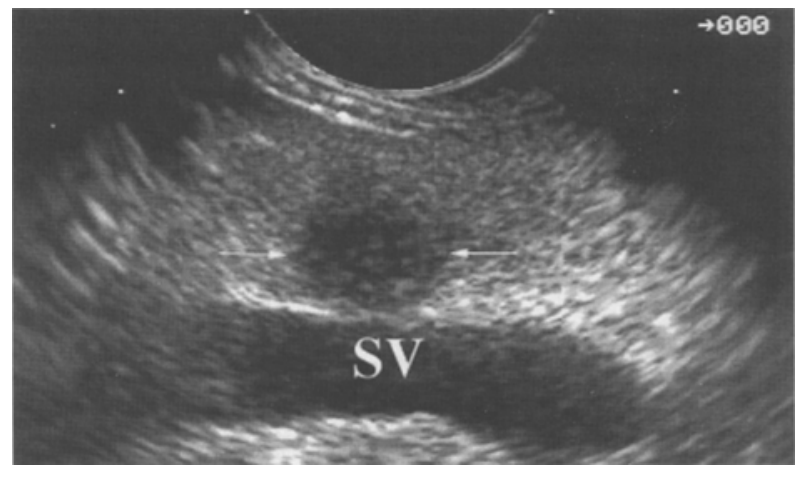

Fig. 7. A 9-mm round hypoechoic, but solid mass (between white arrows) in the neck of the pancreas adjacent to the splenic vein (SV). This nonfunctioning islet cell tumor was found incidently with ultrasound and was resected.

gastrinomas, EUS was found to provide the correct localization in $41 \%$ of patients. When EUS was used in conjunction with endoscopy, the accuracy rate of EUS was $60 \%$. The sensitivity of EUS was $50 \%$ for detecting duodenal gastrinomas and $75 \%$ for pancreatic gastrinomas. EUS diagnosed involved lymph nodes in $62.5 \%$, compared to $0 \%$ with CT scanning (45). EUS can also be used to localize gastrinomas in patients with normal serum gastrins (46).

\section{Chronic Pancreatitis}

EUS imaging of chronic pancreatitis often reveals a large number of changes in the pancreas (Fig. 8). The detailed imaging of the entire pancreas may demonstrate focal or diffuse changes in the parenchyma, ducts, or the presence of intrapancreatic pseudocysts. Early changes in the parenchyma consist of focal areas of increased and/or decreased echo texture of the gland. In more advanced cases, there will be calcifications, lobulations, and bands of fibrosis.

The development of diagnostic EUS criteria for chronic pancreatitis has been hampered by the lack of an acceptable "gold standard" for the diagnosis of chronic pancreatitis. Since good histologic examinations of the pancreas are difficult to obtain, clinicians have been forced to use pancreatography or pancreatic function tests as the basis for the diagnosis of chronic pancreatitis. The specificity and reliability of early ductal changes in pancreatography, when used in making the diagnosis of chronic pancreatitis, have been questioned. Pancreatic function tests may 


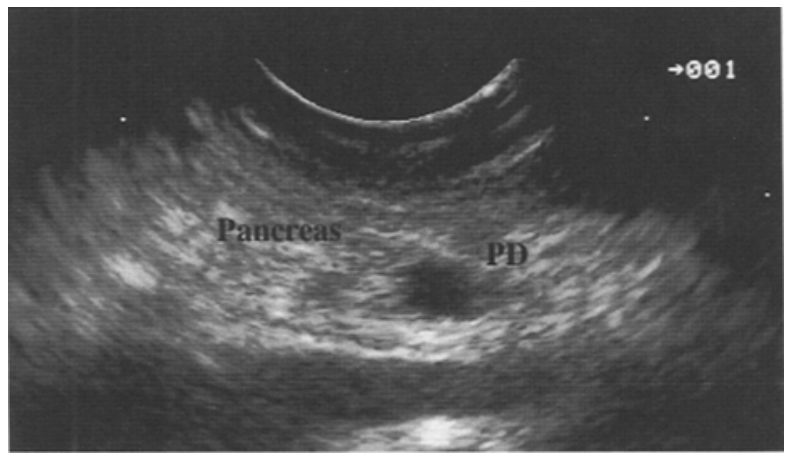

Fig. 8. A thin, fibrotic pancreas associated with a dilated main pancreatic duct (PD). The finding of a heterogenous hyperechoic parenchyma is strongly suggestive of chronic pancreatitis.

also be unreliable for making the diagnosis of chronic pancreatitis with false-positive and false-negative results. Several criteria are used to diagnose chronic pancreatitis by EUS. Most of the individual criteria are not specific enough to make the diagnosis of chronic pancreatitis, and Wiersema et al. have proposed the use of at least three to make the diagnosis of chronic pancreatitis (47). The following ductal changes are seen in chronic pancreatitis: narrowing, dilation, contour changes, duct wall echogenicity, calculi, and side branch dilation. Parenchyma changes include: changes in gland size, focal decreases in parenchymal echogenicity, presence of echogenic foci, cysts, and accentuation of lobular pattern (47). Using these finding in chronic pancreatitis, EUS may be a very sensitive test $(48,49)$.

These findings in the parenchyma and ducts have varying predictive values. The finding of pancreatic calcifications with EUS was $100 \%$ predictive for the diagnosis of chronic pancreatitis. Changes in the pancreatic ducts were predictive of chronic pancreatitis in approx $80 \%$ of cases. The findings of an accentuation of the lobular pattern had the lowest positive predictive rate, $66 \%$. However, differentiating between benign and malignant pancreatitic duct strictures remains problematic (50).

Since EUS is extremely sensitive for the presence of pancreatic cysts and fluid collections, it is very likely that EUS will become the test of choice for demonstrating cystic changes in chronic pancreatitis $(51,52)$. However, there has been no direct comparison with US, CT, and ERCP in patients with chronic pancreatitis. EUS can localize and characterize pseudocysts in detail, and this may be important in cases where cyst aspiration or drainage is planned. Small thin-walled cysts without debris or blood in the fluid are ideal for EUS-guided aspirations or drainage. Some intrapancreatic cysts can be traced back to a large duct or areas of ductal obstruction, and may enlarge with secretin stimulation. Infected pseudocysts or fluid collections can also be diagnosed by sending the aspirated fluid for culture.

\section{References}

1 Giovanni M, Seitz JF. Endoscopic ultrasonography with a linear-type echoendoscope in the evaluation of 94 patients with pancreatobiliary disease. Endoscopy 1994;26:579-585.

2 Rösch T. Endoscopic ultrasonography in pancreatic cancer. Endoscopy 1994; 26: 8066-8067.

3 Yasuda K, Mukai H, Fujimoto S, Kakajima M, Kawai K. The diagnosis of pancreatic cancer by endoscopic ultrasonography. Gastrointest Endosc 1988; 34: 1-8.

4 Snady H, Cooperman A, Siegel J. Endoscopic ultrasonography compared with computed tomography with ERCP in patients with obstructive jaundice or small peripancreatic mass. Gastrointest Endosc 1992: 38: 27-34.

5 Palazzo L, Roseau G, Gayet B, Vilgrain V, Belghiti J, Fekete F, Paolaggi JA. Endoscopic ultrasonography in the diagnosis and staging of pancreatic adenocarcinoma. Results of a prospective study with comparison to ultrasonography and CT scan. Endoscopy 1993; 25: 143-150.

6 Ariyama J, Suyama M, Ogawa K. The detection and prognosis of small pancreatic carcinoma. Int J Pancreatology 1990; 7: 34-47.

7 Muller MF, Meyenberger C, Bertschinger P, Schaer R, Marincek B. Pancreatic tumors: evaluation with endoscopic US, CT, and MR imaging. Radiology 1994; 190: 745-751.

8 Nakaizumi A, Uehara H, Iishi H, Tatsuta M, Kitamura T, Kuroda C, Ohigashi H, Ishikawa O, Okuda S. Endoscopic ultrasonography in diagnosis and staging of pancreatic cancer. Dig Dis Sci 1995; 40: 696-700.

9 Kaufman AR, Sivak MV Jr. Endoscopic ultrasonography in the differential diagnosis of pancreatic disease. Gastrointest Endosc 1989; 35; 214-219.

10 Palazzo L, Roseau G, Gayet B, Vilgrain V, Belghiti J, Fekete F, Paolaggi JA. Endoscopic ultrasonography in the diagnosis and staging of pancreatic adenocarcinoma. Results of a prospective study with comparison to ultrasonography and CT scan. Endoscopy 1993; 25: 143-150.

11 Zerbi A, Balzamo G, Betteru R. Reliability of pancreatic cancer staging classifications. Int J Pancreatology 1994; 15: $13-18$.

12 Tio TL, Tytgat GN, Cikot RJ, Houthoff HJ, Sars PR. Ampullopancreatic carcinoma: preoperative TNM classification with endosonography. Radiology 1990; 107:366-371,

13 Tio TL, Sie LH, Tytgat GN. Endosonography and cytology in diagnosing and staging pancreatic body and tail carci- 
noma. Preliminary results of endosonographic guided puncture. Dig Dis Sci 1993; 38: 59-64.

14 Yasuda K. Mukai H, Nakajima M, Kawai K. Staging of pancreatic carcinoma by endoscopic ultrasonography. Endoscopy 1993; 25: 51-55.

15 Rösch T, Braig C, Gain T, Feuerbach S, Siewert JR, Schusdziarra V, Classen M. Staging of pancreatic and ampullary carcinoma by endoscopic ultrasonography. Comparison with conventional sonography, computed tomography, and angiography. Gastroenterology 1992; 102: 188-199.

16 Snady H, Bruckner H, Siegel J, Cooperman A, Neff R, Kiefer L. Endoscopic ultrasonographic criteria of vascular invasion by potentially resectable pancreatic tumors. Gastrointest Endosc 1994; 40: 326-333.

17 Brugge WR, Lee MJ, Kelsey PB, Schapiro R, Warshaw A. The use of EUS to diagnose malignant portal venous system invasion by pancreatic cancer. Gastrointest Endosc 1996; 43: $561-568$.

18 Mukai H, Nakajima M, Yasuda K, Cho E, Mizuma Y, Hayakumo T, Ashihara T, Mizuno S, Hirano S, Ikeda E. Preoperative diagnosis and staging of pancreatic cancer by endoscopic ultrasonography - a comparative study of other diagnostic tools. Jpn J Gastroenterol 1991; 88: 2132-2142.

19 Smits NJ, Reeders JW. Current applicability of duplex Dopler ultrasonography in pancreatic head and biliary malignancies. Baillieres Clin Gastroenterol 1995; 9: 153-172.

20 Wiersema MJ, Chak A, Kopecky KK, Wiersema LM. Duplex Doppler endosonography in the diagnosis of splenic vein, portal vein, and portosystemic shunt thrombosis. Gastrointest Endosc 1995; 42: 19-25.

21 Jaffe DL, Brugge WR. Endoscopic ultrasonography is useful in evaluating isolated gastric varices. Gastrointest Endosc 1995; 41: 307.

22 Brugge WR, Lee MJ, Warshaw AL. Pancreatic cancer staging: EUS criteria for vascular invasion. Gastrointest Clin North Am 1995; 5: 741-753.

23 Vilman P, Jacobsen GK, Henriksen FW, Hancke S. Endoscopic ultrasonography-guided fine needle aspiration biopsy in pancreatic disease. Gastrointest Endosc 1992;38: 172-173.

24 Vilman P, Hancke S, Henriksen FW, Jacobsen GK. Endoscopic ultrasonography-guided fine-needle aspiration biopsy of lesions in the upper gastrointestinal tract. Gastrointest Endosc 1995; 41: 230-235.

25 Wiersema JM, Wiersema LM, Khurso Q, Cramer HM, Tao LC. Combined endosonography and fine-needle aspiration cytology in the evaluation of gastrointestinal lesions. Gastrointest Endosc 1994; 40: 199-206.

26 Cheng KJ, Katz KD, Durbin TE. Endoscopic ultrasoundguided fine needle aspiration. Gastrointest Endosc 1994; 40: 694-699.

27 Giovannini M, Seitz J.-F, Monges G, Perrier H, Rabbia I. Fine-needle aspiration cytology guided by endoscopic ultrasonography: results in 141 patients. Endoscopy 1995; 27: 171-177.

28 Chang KJ, Albers CG, Nguyen P. Endoscopic ultrasoundguided find needle aspiration of pleural and ascitic fluid. Am J Gastroenterol 1995; 90: 118-150.
29 Tio TL, Sie LH, Tytgat GN. Endosonography and cytology in diagnosing and staging pancreatic body and tail carcinoma. Preliminary results of endosonographic guided puncture. Dig Dis Sci 1993; 38: 59-64.

30 Wiersema M, Wiersema L, Sandusky D, Carr R, Erdel W, Fredrick P. Cost effectiveness of EUS with fine needle aspiration biopsy in evaluating patients with pancreatic malignancy. Gastrointest Endosc 1995; 41: 315.

31 Wiersema M, Sandusky D, Carr R, Erdel W, Fredrick P. Endosonography guided celiac plexus neurolysis in patients with pain due to intra-abdominal malignancy. Gastrointest Endosc 1995; 41: 315.

32 Lawson JM, Baillie J. Endoscopic therapy for pancreatic pseudocysts. Gastrointest Clin North Am 1995; 5: 181-193.

33 Grimm H, Binmoeller KF, Soehendra N. Endosonographyguided drainage of a pancreatic pseudocysts. Gastrointest Endosc 1992; 38: 170-171.

34 Binmoeller KF, Seifer H, Walter A, Soehendra N. Transpapillary and transmural drainage of pancreatic pseudocysts. Gastrointest Endosc 1995; 42: 219-224.

35 Nickl NJ, Lawson JM, Cotton PB. Mucinous pancreatic tumors: ERCP findings. Gastrointest Endosc 1991; 37: 199-201.

36 Raijman I, Kortan P, Walden D. Mucinous ductal ecstasia: cholangiopancreatographic and endoscopic findings. Endoscopy 1994; 26: 303-307.

37 Levy M, Levy P, Hammel P Diagnosis of cystadenomas and cystadenocarcinomas of the pancreas. Gastro Clinique et Biologique 1995; 19: 189-196.

38 Warshaw AL, Compton CC, Lewandrowski K, Cardenosa G, Mueller PR. Cystic tumors of the pancreas: new clinical, radiologic, and pathologic observations in 67 patients. $A n n$ Surg 1990; 212: 432-443.

39 Kato T, Tsukamoto Y, Naitoh Y. Ultrasonographic and endoscopic ultrasonographic angiography in pancreatic mass lesions. Acta Radiologica 1995; 36: 381-387.

40 Glover JR, Shorvon PJ, Lees WR. Endoscopic ultrasound for localization of islet cell tumors. Gut 1992; 33: 108-110.

41 Rösch T, Lightdale CH, Botet JF. Localization of pancreatic endocrine tumors by endoscopic ultrasonography. NEngl J Med 1992; 326: 1721-1726.

42 Rösch T, Classen M. Preoperative localization of endocrine tumors of the pancreas. Gastrointest Clin North Am 1992; 2: 685-695.

43 Zimmer R, Ziegler K, Bader M. Localization of neuroendocrine tumors of the upper GI tract. Gut 1994; 35: 471-475.

44 Lightdale CJ, Botet JF, Woodruff JM, Brennan MF. Localization of endocrine tumors of the pancreas with endoscopic ultrasound. Cancer 1991; 68: 1815-1820.

45 Rosziewship, Amoyal P, Amoyal G. Localization of gastrinomas by endoscopic ultrasonography in patients with Zollinger Ellison syndromes. Surgery 1995; 117: 629-635.

46 Zimmer R, Stolzel U, Bader M, Fett U, Hans-Dieter F, Riecken E, Rehfeld J, Weidenmann B. Brief Report: A duodenal gastrinoma in a patient with diarrhea and normal serum gastrin concentrations. NEJM 1995; 333: 634-636.

47 Wiersema MJ, Hawes RH, Lehman GA, Kochman ML, Sherman S, Kopecky KK. Prospective evaluation of endoscopic ultrasonography and endoscopic retrograde 
cholangiopancreatography in patients with chronic abdominal pain of suspected pancreatic origin. Endoscopy 1993; 29: 555-564.

48 Buscail L, Escourrou J, Moreau J, Delvaux M, Louvel D, Lapeyre F, Tregant P, Frexinos J. Endoscopic ultrasonography in chronic pancreatitis: a comparative prospective study with conventional ultrasonography, computed tomography and ERCP. Pancreas 1995; 10: 251-257.

49 Natterman C, Goldschmidt AJ, Dancygier H. Endosonography in chronic pancreatitis-a comparison between endoscopic retrograde pancreatography and endoscopic ultrasonography. Endoscopy 1993; 25: 565-570.
50 Furukawa T, Tsukamoto Y, Naitoh Y, Hirooka V, Hayakawa T. Differential diagnosis between benign and malignant localized stenosis of the main pancreatic duct by intraductal ultrasound of the pancreas. Am J Gastroenterol 1994; 89: 2038-2041.

51 Ikeda M, Sato T, Ochiai M, Morozumi A, Ainota T, Fujino MA. Ultrasonographic follow-up study of small pancreatic cysts of unknown etiology. Bildgebung 1993; 60: 209-214.

52 Ikeda M, Sato T, Ochiai M, Morozumi A, Nakamura T, Fujino MA. Morphological changes of small pancreatic cysts in response to secretin stimulation. Observation by endoscopic ultrasonography. Dig Dis Sci 1993; 38: 648-652. 